\title{
A Remarkable and Durable Response to Sintilimab and Anlotinib in the First-Line Treatment of an Anaplastic Thyroid Carcinoma without Targetable Genomic Alterations: A Case Report
}

\author{
Lin Gui ${ }^{1}$ \\ Shaoyan Liu ${ }^{2}$ \\ Ye Zhang ${ }^{3}$ \\ Yuankai Shi $\mathbb{D}^{\prime}$ \\ 'Department of Medical Oncology, \\ National Cancer Center/National Clinical \\ Research Center for Cancer/Cancer \\ Hospital, Chinese Academy of Medical \\ Sciences \& Peking Union Medical College, \\ Beijing Key Laboratory of Clinical Study \\ on Anticancer Molecular Targeted Drugs, \\ Beijing, People's Republic of China; \\ ${ }^{2}$ Department of Head and Neck Surgery, \\ National Cancer Center/National Clinical \\ Research Center for Cancer/Cancer \\ Hospital, Chinese Academy of Medical \\ Sciences and Peking Union Medical \\ College, Beijing, People's Republic of \\ China; ${ }^{3}$ Department of Radiation \\ Oncology, National Cancer Center/ \\ National Clinical Research Center for \\ Cancer/Cancer Hospital, Chinese \\ Academy of Medical Sciences and Peking \\ Union Medical College, Beijing, People's \\ Republic of China
}

Correspondence: Yuankai Shi Department of Medical Oncology, National Cancer Center/National Clinical Research Center for Cancer/Cancer Hospital, Chinese Academy of Medical Sciences \& Peking Union Medical College, Beijing Key Laboratory of Clinical Study on Anticancer Molecular Targeted Drugs, No. 17, Panjiayuan Nanli, Chaoyang

District, Beijing, I0002 I, People's

Republic of China

Tel +86-10-87788293

Fax +86-10-87778740

Email syuankai@cicams.ac.cn

\begin{abstract}
Anaplastic thyroid carcinoma (ATC) is a rare and highly aggressive fatal tumor. Most ATC patients using traditional surgery or radio-chemotherapy have poor prognosis and experience recurrence in a very short time. There is no optimal therapy for ATC, and the median survival time is about 5 months. We report a 67 -year-old ATC patient, who experienced rapid local recurrence after radical thyroidectomy. The resected tumor tissue was sent for immunohistochemistry analysis and targeted next-generation sequencing. The results indicated high PD-L1 expression, a tumor mutation burden of 0.48 muts/Mb, microsatellite stable, and somatic mutations of TERT promoter, EIF1AX, NRAS and TP53. However, none of the mutations indicated corresponding target therapy. An immediate operation was unsuitable because of rapid recurrence after surgery. The patient was also not in a condition to tolerate chemotherapy. Based on the high expression of PD-L1, an optimum strategy was used, combining immunotherapeutic agent, sintilimab, with an antiangiogenesis drug, anlotinib. The patient obtained remarkable tumor shrinkage and an 18.3-month-sustained remission period. This is an effective case of using immunotherapy and anti-angiogenesis agent in the first-line treatment of ATC. It demonstrates a feasible and novel therapeutic option for future treatment of ATC patients.
\end{abstract}

Keywords: anaplastic thyroid carcinoma, PD-1 inhibitor, anti-angiogenesis drug, TERT promoter mutations, TP53 mutations, NRAS mutation, next-generation sequencing

\section{Introduction}

Anaplastic thyroid carcinoma (ATC) is a rare and highly fatal malignancy with characteristic rapid progression, early metastasis, and poor prognosis, accounting for about $1-2 \%$ of all thyroid cancers. ${ }^{1}$ ATC tumor comprises heterogeneous mixtures of spindle-shaped, epithelioid, and pleomorphic giant cells. Histopathological features of ATC include tumor necrosis, obvious nuclear pleomorphism, and high frequency of atypical mitosis. Major metastases usually occur in the lungs, followed by bones, intrathoracic and neck lymph nodes. ${ }^{2,3}$ ATC is most prevalent in the 60-70-year age group, and its frequency is higher in females than males $(2: 1.5){ }^{1}$

Traditional clinical management of ATC involves surgery, radiotherapy and/or chemotherapy. ${ }^{4}$ However, these approaches often do not control the disease because many patients are diagnosed with inoperable or distant metastases. ${ }^{4}$ The 
disease-specific mortality rate is nearly $100 \%$, and the median survival time, after diagnosis, is approximately 5 months; only $20 \%$ of patients survive more than one year. ${ }^{1}$ Therefore, it is urgent to establish novel diagnostic and standard strategies for ATC.

Multiple driver mutations of ATC have been identified with the development of next-generation sequencing, such as oncogenic mutations of MAPK pathway (BRAF, RAS), mutations in TP53 and the TERT promoter, and fusions in $R E T$ and $N T R K .^{5}$ Dabrafenib, a selective inhibitor of $B R A F$ mutant, and trametinib, a selective MEK inhibitor, were approved by the US Food and Drug Administration (FDA) in 2018, for the treatment of ATC patients with $B R A F$ mutation. Other studies have shown that PD-L1 was exclusively expressed on ATC tumor cells, and T cells infiltrated the microenvironment. ${ }^{6}$ As such, several novel monoclonal antibodies, such as nivolumab, pembrolizumab and sintilimab, are under clinical trials and are proposed to be highly promising treatment modalities. ${ }^{5}$ It has been reported that immunotherapy combined with antiangiogenic drugs have synergistic anti-tumor activity, because the drugs could promote $\mathrm{T}$ cell infiltration. ${ }^{7}$ Multitarget tyrosine kinase inhibitor (MTKI) anlotinib, a novel anti-angiogenesis agent, is a popular drug for its efficacy against multiple cancers and low side effects. Previous reports showed that anlotinib could effectively inhibit ATC cell lines. ${ }^{8}$ We reported an ATC patient who had recurrence after radical surgery, having TP53 and NRAS mutation, and high expression of PD-L1, but not $B R A F$ mutations. PD-1 inhibitor, sintilimab, and antiangiogenesis drug, anlotinib, were used, and major remission (about 18 months) was obtained. This report was the first case that investigated and proved the efficacy of immunotherapy combined with anti-angiogenesis agent for ATC, but it still needs a larger investigation.

\section{Case Presentation}

A 67-year-old female presented with the chief complaint of rapidly growing neck mass and hoarseness. Contrastenhanced computed tomography (CT) revealed a neoplastic lesion of $5 \mathrm{~cm}$ in the left lobe of thyroid gland and multiple small lymph nodes in the neck but no distant metastases. A surgery was performed on March 20, 2019. The tumor was found to be closely related to muscles and esophagus. The pathology of intraoperative freezing indicated high-grade malignant tumor. She underwent total thyroidectomy and central neck lymph node dissection. The surgical pathology showed multifocal, bilateral anaplastic thyroid carcinoma (ATC). The tumors broke through the thyroid capsule and involved the surrounding muscles, and the left central lymph nodes were involved (pT4N1M0, IVB). The immunohistochemistry (IHC) was Ki-67 (80\% positive), EGFR, P53, AE1/AE3, P16 positive, and TG, P40, EBR, TTF-1, P63, LCA negative. Eleven days later, she underwent a debridement because of esophageal leakage. Unfortunately, two weeks after the second surgery, the patient had a feeling of suffocation. A mass with rapidly progressive enlargement was observed in the operated area with a range of $6.7 \times 6.5 \times 2.8 \mathrm{~cm}$ on magnetic resonance imaging (MRI), and was thought to be tumor recurrence. Multiple metastatic lymph nodes were found in the bilateral neck, the biggest one about $1.6 \times 1.0 \mathrm{~cm}$ (Figure 1A). The patient was referred to the Cancer Hospital, Chinese Academy of Medical Sciences \& Peking Union Medical College. The patient had a poor Eastern Cooperative Oncology Group (ECOG) performance status of 3 with difficulty in breath, unable to lie flat, swollen face and hoarse voice. So, she could not tolerate chemotherapy, radiotherapy or an immediate operation.

Tumor tissue from the thyroidectomy was sent to Genetron Health (Beijing) for molecular testing and immunohistochemical analysis (IHC) of programmed death-ligand 1 (PD-L1). DNA from a paraffin-embedded sample of ATC and the patient's peripheral blood (as control) were analyzed with the Onco PanScan ${ }^{\mathrm{TM}}$ (Genetronhealth, Beijing, China), which interrogates 825 cancer-related genes by next-generation sequencing (NGS). The results revealed a tumor mutation burden of 0.48 muts $/ \mathrm{Mb}$, microsatellite stable (MSS), and somatic mutations of TERT promoter, EIF1AX, NRAS and TP53 (Table 1). PD-L1 IHC 22C3 pharmDx assay (Agilent Technologies, Carpinteria, CA, USA) was used to assess the expression of PD-L1 in formalin-fixed thyroid tumor samples, evaluated by both the tumor proportion score (TPS) and the combined positive score (CPS). At least 100 tumor cells must be present in the sample for evaluation. The tumor PD-L1TPS was $60 \%$ and CPS was 70 .

The patient was unsuitable for an immediate operation as subsequent treatment given that recurrence occurred shortly after surgery. Also, the patient was not in a condition to tolerate chemo- or radio-therapy. Sintilimab, an immune checkpoint inhibitor, was suggested as an effective treatment option because of the identified association between high PD-L1 expression (TPS $\geq 50 \%$ ) and improved responses to immune 


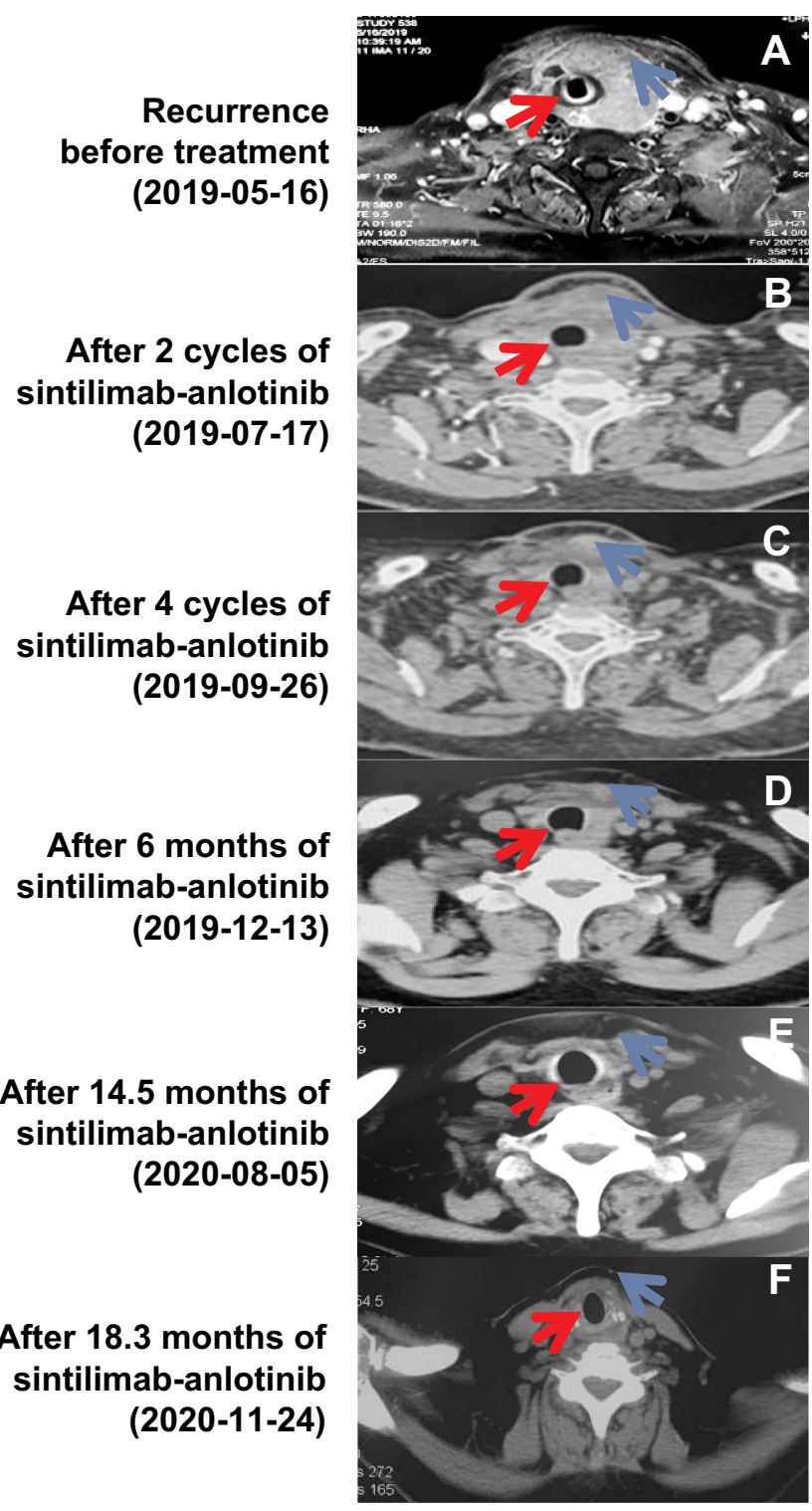

Figure I Radiological monitoring of the patient before and after treatment with sintilimab plus anlotinib. (A) Magnetic resonance imaging (MRI) was performed on the recurrence before treatment (Baseline), on May 16, 2019. (B and C). Computerized tomography (CT) on July 17, 2019 (B) and September 26, 2019 (C) showed the tumor shrank after 2 and 4 cycles of sintilimab-anlotinib, respectively. (D) CT on December 13, 2019 identified a little residue of the recurrence mass. (E) CT after 14.5 months of the treatment showed a sustained and prominent reduction of the tumor. (F) The tumor shrinkage was continued over 18.3 months as shown by the CT on November 24, 2020. The red arrow indicates tracheal compression, whereas the blue arrow indicates the tumor.

checkpoint inhibitors. Furthermore, it was proved that anti-angiogenesis drug could improve the immunotherapeutic response; thus, an anti-angiogenesis drug, anlotinib, was added. Starting from June 6, 2019, the immunotherapy agent sintilimab was given $200 \mathrm{mg}$ every 3 weeks, combined with anti-angiogenesis drug, oral anlotinib $12 \mathrm{mg}$, once daily, 2-week on/1-week off. After 2 cycles (6 weeks) of treatment, CT scanning showed significant
Table I NGS* Gene Mutation Profiling in Primary Thyroid Lesion

\begin{tabular}{|l|c|c|c|c|}
\hline Gene & Mutation Type & Genovariation & $\begin{array}{c}\text { Amino } \\
\text { Acid } \\
\text { Variation }\end{array}$ & $\begin{array}{c}\text { Allelic } \\
\text { Frequency }\end{array}$ \\
\hline EIFIAX & Missense mutation & C.25G>C & P. G9R & $7.2 \%$ \\
NRAS & Missense mutation & c. $182 \mathrm{~A}>\mathrm{G}$ & P. Q6IR & $11.0 \%$ \\
TP53 & Missense mutation & C.584T>C & P. II95T & $19.7 \%$ \\
TERT & Upstream promoter & C228T & $/$ & $22.58 \%$ \\
& mutation & & & \\
\hline
\end{tabular}

Abbreviation: *NGS, next-generation sequencing.

reduction of the mass in the neck, with a maximum crosssectional area (MCSA) shrinking from $6.7 \times 6.5 \mathrm{~cm}$ to $3.7 \times 3.6 \mathrm{~cm}$. Metastatic lymph nodes in the left neck were narrowed, with a short diameter shrinking from $1.0 \mathrm{~cm}$ to $0.7 \mathrm{~cm}$ in the largest one, and no swelling on the right side (Figure 1B). Partial response was achieved according to RECIST v1.1. In addition, the mass continued to shrink with treatment (Figure 1C). Six months later, CT scan showed little remains with the MCSA of about $1.9 \times 1.4 \mathrm{~cm}$ (Figure 1D). A follow-up CT scan at 14.5 months and 18.3 months after treatment demonstrated prominent and sustained reduction of the tumor (Figure $1 \mathrm{E}$ and $\mathrm{F}$ ). The esophagus was damaged during surgery (radiation may cause esophagus injury), and the patient was unwilling to undergo consolidation radiotherapy. Grade 1 rash was the only adverse event observed during treatment. Patient-reported outcome surveys, at approximately 18.3 months of treatment, indicated an excellent quality of life, including continued normal physical exercises such as swimming and jogging.

\section{Discussion}

ATC is one of the most fatal cancers. The median survival time after diagnosis is only about 5 months. ${ }^{9}$ Currently, surgery is one of the common treatments for ATC. However, majority of patients with ATC at diagnosed are distant metastasis or locally advanced, which are not amenable to completing resection. ${ }^{1,10}$ Besides, anaplastic carcinoma surgical intervention is affected by more severe complication, including recurrent laryngeal nerve (RLN) palsy, ${ }^{11}$ hypocalcemia ${ }^{12}$ and lymph nodal involvement. ${ }^{13}$ Majority of ATC patients have recurrence soon after surgery. Thus, the benefit of surgical treatment to patient survival is limited. The patient reported here relapsed shortly after surgery, having a poor condition with ECOG score of 3, all of which showed no indication to 
commence chemotherapy or radiotherapy. Precise treatment guided by gene diagnosis to determine the efficacy of targeted therapies, such as BRAF inhibitor for $B R A F$ $V 600 E$ mutant positive patients ${ }^{14}$ and TRK inhibitor for NTRK fusion positive patients ${ }^{15}$ and inhibitors for RET or ALK rearrangement patients, ${ }^{16,17}$ may be an appropriate strategy. However, NGS results of this patient did not show any mutations or rearrangements that had corresponding targeted therapeutic agents; therefore, multitarget anti-angiogenic drugs were considered. Recently, Multi-target tyrosine kinase inhibitor (MTKI) lenvatinib was approved by FDA for the management of advanced differential thyroid cancer after radioactive iodine treatment, and also proposed for ATC patients who do not have other effective therapeutic options. However, the effective rate of lenvatinib for ATC patients was only $17-24 \%{ }^{18}$ Anlotinib is a novel MTKI and antiangiogenic drug that can inhibit platelet-derived growth factor receptors, Ret, Aurora-B, epidermal growth factor receptors (EGFR), and fibroblast growth factor receptor. ${ }^{8}$ Anlotinib showed anticancer activity on ATC cell lines. It can inhibit tumor cell proliferation by causing ATC cells to have abnormal spindle assembly, G2/M phase arrest, TP53 activation, thereby suppressing cell migration and growth of xenograft thyroid tumors by disturbing the formation of F-action. ${ }^{8}$ According to current clinical reports, anlotinib is safe and effective in the treatment of advanced refractory thyroid cancer. ${ }^{19}$ For 58 patients with locally advanced or metastatic medullary thyroid cancer who received anlotinib, $56.9 \%$ had a partial response, and the progression-free survival rate at 48 weeks was $85.5 \%{ }^{20}$ Therefore, anlotinib potentially offers an effective treatment option for ATC patients.

The patient had high PD-L1 expression (TPS 60\%, CPS 70). Meta-analysis in 2018 showed that PD-L1 overexpression in patients with non-medullary thyroid cancer is significantly associated with poor survival, disease recurrence and concurrent thyroiditis. ${ }^{21}$ This suggests that PD-L1 may be a prognostic biomarker, and anti-PD $-1 / \mathrm{PD}-\mathrm{L} 1$ immunotherapy may have promise in the PD-L1 positive locally advanced, unresectable, or metastatic thyroid cancer patients. PD-L1 IHC positivity has been implicated as a predictive biomarker in PD-1/PD-L1 checkpoint blockade immunotherapy in a variety of malignancies. It was reported that BRAF V600E mutation and PD-L1 expression $(3+)$ were identified in the tumor tissue of a $62-$ year-old male ATC patient, with local thyroid recurrence, distant metastases after initial surgery and no recovery after first- and second-line chemotherapies. ${ }^{22}$ Subsequent treatment with vemurafenib and nivolumab led to substantial regression of tumor nodules. A distinct decrease in metastatic lesions and complete remission was observed 20 months after initiation of nivolumab, demonstrating the effectiveness of immunotherapy for this patient. And an initially unresectable, BRAF-mutated ATC patient with a PD-L1 TPS of $95 \%$ was treated with dabrafenib, trametinib, and pembrolizumab. A meaningful response was achieved and the tumor was completely resected. MD Anderson cancer center retrospectively reviewed 12 ATC patients treated with pembrolizumab in combination with kinase inhibitor at the time of disease progression; $42 \%$ had a partial response and $33 \%$ had stable disease; pembrolizumab was considered an effective salvage treatment. ${ }^{23}$ Besides, previous studies showed that melanoma patients with NRAS mutations in tumor had better response to immunotherapy and better outcomes than patients whose tumors had other genetic subtypes. ${ }^{24}$ Here, the ATC patient had mutation of NRAS, which may be one of the reasons why the patient was suitable for immunotherapy. Currently, several clinical trials on PD-1/ PD-L1 inhibitors for ATC, as single or combination therapy strategies, are ongoing, including NCT02688608, NCT03211117, NCT03246958, and NCT03181100. Sintilimab is a highly selective, humanized, monoclonal antibody that blocks the interaction between PD-1 and its ligands. The agent was approved for the treatment of relapsed or refractory classical Hodgkin lymphoma in China, and it has been tested on multiple advanced solid tumors. ${ }^{25}$ Therefore, PD-1/PD-L1 immunotherapy using sintilimab was suitable for this patient.

It was recently found that many cancer patients could not benefit from immunotherapy alone, due to vascular abnormalities that facilitate immune evasion in most solid tumors. Judicious use of drugs targeting proangiogenic factors (VEGF, bFGF, ${ }^{16,17}$ PDGF) and their receptors (VEGFR, FGFR, PDGFR) can improve therapeutic response. This is likely due to the normalization of tumor vasculature, therefore increasing the infiltration of immune cells and improving the immunosuppressed microenvironment. ${ }^{7}$ Many studies have evaluated the combination of immune checkpoint inhibitors with the anti-angiogenic drug, bevacizumab, across various tumor types, and most demonstrated marked improvement in the objective response rate, progression-free survival and overall survival. ${ }^{26}$ Similarly, the antitumor activity of lenvatinib plus anti-PD-1 antibodies was greater than that of either single agent. ${ }^{27}$ Several clinical trials with 
these combined treatments are being tested for thyroid cancer, but no conclusions have been reached. MD Anderson cancer center is studying the efficiency of lenvatinib and pembrolizumab for the treatment of stage IVC metastatic ATC, in an ongoing clinical trial NCT04171622. The National Cancer Institute (NCI) is evaluating the effect of lenvatinib and pembrolizumab combined treatment on differentiated thyroid cancer (DTC), in the NCT02973997 trial. NCI is also testing the effectiveness of the combination of MTKI agent cabozantinib, nivolumab and ipilimumab for advanced DTC, in the NCT03914300 trial. In this study, the tumor shrunk remarkably on treatment with the antiangiogenic drug anlotinib combined with the immunotherapeutic agent sintilimab, and the clinical benefit for the ATC patient has continued over 18.3 months. This case provides evidence of the effectiveness of this combination in ATC patients.

\section{Conclusion}

This case reported an ATC patient, who had recurrence within a short period after radical surgery, had high expression of PD-L1, and TP53 and NRAS mutation, but was TMB-low, MSS and did not have genomic alterations with level 1 to 4 of evidence according to the OncoKB knowledge database. PD-1 inhibitor, sintilimab, along with anti-angiogenesis drug, anlotinib, were administrated to the patient. Major and sustained remission was obtained. This report was the first case that investigated and proved the efficacy of immunotherapy combined with antiangiogenesis agent in the first-line treatment of ATC, and the regimen is worthy of further study in ATC.

\section{Ethics Approval}

The study was approved by the Institutional Review Board of the Cancer Institute/Hospital, Chinese Academy of Medical Sciences \& Peking Union Medical College.

\section{Consent to Publication}

Written informed consent for the publication of this case details was obtained from the patient.

\section{Acknowledgments}

We thank Genetron Health (Beijing, China) for conducting genetic testing and immunohistochemistry analysis. Genetic writing support was also provided by Genetron Health (Beijing, China).

\section{Author Contributions}

All authors made a significant contribution to the work reported, whether that is in the conception, study design, execution, acquisition of data, analysis and interpretation, or in all these areas; took part in drafting, revising or critically reviewing the article; gave final approval of the version to be published; have agreed on the journal to which the article has been submitted; and agree to be accountable for all aspects of the work.

\section{Funding}

No funding was received for conducting this study.

\section{Disclosure}

The authors declare no competing interests.

\section{References}

1. Molinaro E, Romei C, Biagini A, et al. Anaplastic thyroid carcinoma: from clinicopathology to genetics and advanced therapies. Nat Rev Endocrinol. 2017;13(11):644-660. doi:10.1038/nrendo.2017.76

2. Rodriguez JM, Piñero A, Ortiz S, et al. Clinical and histological differences in anaplastic thyroid carcinoma. Eur J Surg. 2000;166 (1):34-38. doi:10.1080/110241500750009672

3. Rühli FJ, Hilfiker PR. Metastasis of thyroid cancer to the heart. AJR Am J Roentgenol. 2001;177(2):474. doi:10.2214/ajr.177.2.1770474

4. Glaser SM, Mandish SF, Gill BS, Balasubramani GK, Clump DA, Beriwal S. Anaplastic thyroid cancer: prognostic factors, patterns of care, and overall survival. Head Neck. 2016;38(Suppl 1):E20832090. doi:10.1002/hed.24384

5. Pozdeyev N, Rose MM, Bowles DW, Schweppe RE. Molecular therapeutics for anaplastic thyroid cancer. Semin Cancer Biol. 2020;61:S1044579X20300067. doi:10.1016/j.semcancer.2020.01.005

6. Bastman JJ, Serracino HS, Zhu Y, et al. Tumor-infiltrating T cells and the PD-1 checkpoint pathway in advanced differentiated and anaplastic thyroid cancer. J Clin Endocrinol Metab. 2016;101(7):2863-2873. doi:10.1210/jc.2015-4227

7. Fukumura D, Kloepper J, Amoozgar Z, Duda DG, Jain RK Enhancing cancer immunotherapy using antiangiogenics: opportunities and challenges. Nat Rev Clin Oncol. 2018;15(5):325-340. doi:10.1038/nrclinonc.2018.29

8. Ruan X, Shi X, Dong Q, et al. Antitumor effects of anlotinib in thyroid cancer. Endocr Relat Cancer. 2019;26(1):153-164. doi:10. 1530/ERC-17-0558

9. Kebebew E, Greenspan FS, Clark OH, Woeber KA, McMillan A Anaplastic thyroid carcinoma. Treatment outcome and prognostic factors. Cancer. 2005;103(7):1330-1335. doi:10.1002/cncr.20936

10. Swift PS, Larson S, Clark OH, Ruan D. 34 - cancer of the thyroid. In: Hoppe RT, Phillips TL, Roach M, editors. Leibel and Phillips Textbook of Radiation Oncology (Third Edition). 3rd ed. W.B. Saunders; 2010:726-736. doi:10.1016/B978-1-4160-5897-7.00035-4

11. Calò PG, Medas F, Conzo G, et al. Intraoperative neuromonitoring in thyroid surgery: is the two-staged thyroidectomy justified? Int J Surg. 2017;41 Suppl 1:S13-S20. doi:10.1016/j.jjsu.2017.02.001

12. Docimo G, Tolone S, Ruggiero R, et al. Total thyroidectomy without prophylactic central neck dissection combined with routine oral calcium and vitamin D supplements: is it a good option to achieve a low recurrence rate avoiding hypocalcemia? A retrospective study. Minerva Chir. 2013;68(3):321-328. 
13. Conzo G, Docimo G, Mauriello C, et al. The current status of lymph node dissection in the treatment of papillary thyroid cancer. A literature review. Clin Ter. 2013;164(4):e343-346. doi:10.7417/ CT.2013.1599

14. Subbiah V, Kreitman RJ, Wainberg ZA, et al. Dabrafenib and trametinib treatment in patients with locally advanced or metastatic BRAF V600-mutant anaplastic thyroid cancer. J Clin Oncol. 2018;36 (1):7-13. doi:10.1200/JCO.2017.73.6785

15. Cocco E, Scaltriti M, Drilon A. NTRK fusion-positive cancers and TRK inhibitor therapy. Nat Rev Clin Oncol. 2018;15(12):731-747. doi:10.1038/s41571-018-0113-0

16. Offin M, Guo R, Wu SL, et al. Immunophenotype and response to immunotherapy of RET-rearranged lung cancers. JCO Precis Oncol. 2019;3. doi:10.1200/PO.18.00386

17. Leroy L, Bonhomme B, Le Moulec S, Soubeyran I, Italiano A, Godbert Y. Remarkable response to ceritinib and brigatinib in an anaplastic lymphoma kinase-rearranged anaplastic thyroid carcinoma previously treated with crizotinib. Thyroid. 2020;30(2):343-344. doi:10.1089/thy.2019.0202

18. Tahara M, Kiyota N, Yamazaki T, et al. Lenvatinib for anaplastic thyroid cancer. Front Oncol. 2017;7:25. doi:10.3389/fonc.2017. 00025

19. Sun Y, Niu W, Du F, et al. Safety, pharmacokinetics, and antitumor properties of anlotinib, an oral multi-target tyrosine kinase inhibitor, in patients with advanced refractory solid tumors. J Hematol Oncol. 2016;9(1):105. doi:10.1186/s13045-016-0332-8

20. Sun Y, Du F, Gao M, et al. Anlotinib for the treatment of patients with locally advanced or metastatic medullary thyroid cancer. Thyroid. 2018;28(11):1455-1461. doi:10.1089/thy.2018.0022
21. Aghajani M, Graham S, McCafferty C, et al. Clinicopathologic and prognostic significance of programmed cell death ligand 1 expression in patients with non-medullary thyroid cancer: a systematic review and meta-analysis. Thyroid. 2018;28(3):349-361. doi:10.1089/thy.20 17.0441

22. Kollipara R, Schneider B, Radovich M, Babu S, Kiel PJ. Exceptional response with immunotherapy in a patient with anaplastic thyroid cancer. Oncologist. 2017;22(10):1149-1151. doi:10.1634/theoncologist.2017-0096

23. Iyer PC, Dadu R, Gule-Monroe M, et al. Salvage pembrolizumab added to kinase inhibitor therapy for the treatment of anaplastic thyroid carcinoma. J Immunother Cancer. 2018;6(1):68. doi:10. 1186/s40425-018-0378-y

24. Muñoz-Couselo E, Adelantado EZ, Ortiz C, García JS, Perez-Garcia J. NRAS-mutant melanoma: current challenges and future prospect. Onco Targets Ther. 2017;10:3941-3947. doi:10.2147/OTT.S117121

25. Hoy SM. Sintilimab: first global approval. Drugs. 2019;79 (3):341-346. doi:10.1007/s40265-019-1066-z

26. Chen DS, Hurwitz H. Combinations of bevacizumab with cancer immunotherapy. Cancer J. 2018;24(4):193-204. doi:10.1097/PPO.00 00000000000327

27. Kato Y, Tabata K, Kimura T, et al. Lenvatinib plus anti-PD-1 antibody combination treatment activates $\mathrm{CD} 8+\mathrm{T}$ cells through reduction of tumor-associated macrophage and activation of the interferon pathway. PLoS One. 2019;14(2):e0212513. doi:10.1371/journal. pone. 0212513
OncoTargets and Therapy

\section{Publish your work in this journal}

OncoTargets and Therapy is an international, peer-reviewed, open access journal focusing on the pathological basis of all cancers, potential targets for therapy and treatment protocols employed to improve the management of cancer patients. The journal also focuses on the impact of management programs and new therapeutic

\section{Dovepress}

agents and protocols on patient perspectives such as quality of life, adherence and satisfaction. The manuscript management system is completely online and includes a very quick and fair peer-review system, which is all easy to use. Visit http://www.dovepress.com/ testimonials.php to read real quotes from published authors. 